\title{
Estudo e Proposta para utilização dos metadados OBAA na descrição de jogos sérios
}

\author{
Vinícius de Carli ${ }^{1}$, Maximiliano Reidel ${ }^{1}$, Maurício Covolan Rosito ${ }^{1}$, \\ Anita Maria da Rocha Fernandes ${ }^{2}$, Júlia Marques Carvalho da Silva ${ }^{1}$ \\ ${ }^{1}$ Instituto Federal do Rio Grande do Sul, Campus Bento Gonçalves \\ Av. Osvaldo Aranha, 540 - Bento Gonçalves, RS - Brasil \\ vinidcali@gmail.com, \{maximiliano.reidel, mauricio.rosito, \\ julia.silva\}@bento.ifrs.edu.br \\ ${ }^{2}$ Universidade do Vale do Itajaí \\ BR 101, Km 207 - MundoCar Shopping, Kobrasol - São José, SC - Brasil \\ vinidcali@gmail.com, \{maximiliano.reidel, mauricio.rosito, \\ julia.silva\}@bento.ifrs.edu.br, anita.fernandes@univali.br
}

\begin{abstract}
Serious games aims to training, educating or simulating some knowledge beyond gamers play. Therefore, they can be used as a learning object. However, learning objects metadata does not support the description of this media. This paper presents a study how serious games can be described as learning objects through OBAA metadata. We analyzed the features of 66 games. Next, a new set of metadata elements and/or values were indicated.

Resumo. Os jogos sérios objetivam o treinamento, ensino ou simulação de algum conhecimento enquanto o jogador os utilizam. Nesta perspectiva, eles podem ser considerados como objetos de aprendizagem. Entretanto, os metadados que descrevem tais objetos de aprendizagem atualmente não suportam esta mídia. Este artigo apresenta um estudo de como os jogos sérios podem ser descritos através dos metadados OBAA. Para isto, foram analisados 66 jogos e então proposto um conjunto de elementos a serem acrescentados.
\end{abstract}

\section{Introdução}

O conceito e uso de objetos de aprendizagem vem se tornando cada vez mais popular. De modo geral, eles consistem em recursos utilizados como instrumento na prática educativa, como: textos, fotos, músicas, vídeos e softwares. Dentro desta perspectiva, cabe também a utilização de jogos. No caso dos jogos digitais, eles apresentam um ambiente controlado e dinâmico (imagens e sons) com regras impostas, que visam criar a sensação de imersão e realismo [Susi et al 2005]. Estes podem ter caráter de entretenimento ou objetivar o ensino ou a prática de algum conhecimento, denominados então de Jogos Sérios. Devido a aplicabilidade pedagógica, os jogos sérios tornam-se também um tipo de objeto de aprendizagem.

As discussões sobre a utilização jogos como objetos de aprendizagem são recentes. Nash (2005) afirma que os jogos sérios consistem em um novo tipo de objeto de aprendizagem, podendo ser incluídos em salas de aula tradicionais ou em cursos online. Segundo Janson e Janson (2009), o objeto de aprendizagem do tipo jogo distinguese de qualquer outro tipo de aplicação didática, pois desafia o aprendiz a alcançar os objetivos propostos. Mediante o crescente interesse em se utilizar jogos como objetos de 
aprendizagem, Torrent et al (2009), por exemplo, desenvolveram uma plataforma que permite o desenvolvimento de jogos sérios como objetos de aprendizagem. Desta forma, assim como qualquer recurso didático, observa-se que a opção de utilização dos jogos sérios recaí sob o professor, cabendo a ele eleger o conteúdo e momento apropriado dentro de seu planejamento pedagógico. Contudo, sob o ponto de vista computacional há de se fazer algumas considerações.

Tecnicamente, um objeto de aprendizagem deve apresentar algumas características como: ser disponibilizado em repositórios, ser empacotado para posterior execução em ambientes virtuais de aprendizagem, e conter um arquivo de metadados responsável pela sua descrição. Tal arquivo deve seguir uma especificação, de modo a ter a descrição identificada corretamente pelos sistemas de informação (repositórios). Atualmente, existem várias especificações, as quais variam e implementam diferentes necessidades. Elas permitem descrever objetos de aprendizagem tradicionais, ou seja, não contemplam características encontradas especificamente em jogos. Dentre as possibilidades, nesta pesquisa optou-se pela especificação OBAA (Objetos de Aprendizagem Baseados em Agentes) devido a característica de fácil expansão a cenários específicos (ex: acessibilidade e segmentação) e recente proposta, a qual contempla tecnologias recentes [Vicari 2010].

Entretanto, a ideia de se desenvolver um conjunto de metadados para jogos sérios já vem sendo discutida. Hendrix et al. (2012) apresentam uma proposta, entretanto não especificam como os elementos podem ser incluídos nas especificações atuais, nem como implementá-la em ferramentas ou repositórios. Diante ao cenário exposto, este artigo visa responder a seguinte questão: "quais metadados são relevantes para a descrição de um jogo sério como objeto de aprendizagem?". Para isto, inicialmente foi realizado um levantamento das características gerais que os jogos apresentam. Em seguida, foi verificado como estas características se configuram nos jogos atuais, onde aqui foi realizado um estudo com 66 jogos. Tal estudo permitiu verificar a aderência e relevância das características, bem como limitações nas descrições. Por fim, é apresentada uma proposta de extensão dos metadados baseado no OBAA.

\section{Jogos Sérios como Objeto de Aprendizagem}

Atualmente, existem vários tipos de classificações de jogos, uma delas refere-se ao objetivo do jogo. Nele, os jogos podem ser considerados como: jogos casuais (casual games), jogos sérios (serious games) e os jogos publicitários (advergames) [Morais 2011]. Os jogos sérios têm como definição o uso do jogo além de apenas entreter o jogador, podendo ser aplicado para treinamento, simular ou educar [Susi et al. 2005]. Ainda, eles podem ser jogados tanto em momentos de lazer ou ser incluído como uma atividade pedagógica dentro de um contexto educativo. O professor pode utilizá-lo em sala de aula, pois a temática do jogo ou habilidades a serem desenvolvidas poderá auxiliar o aluno na aprendizagem de algum conteúdo. Desta forma, o jogo torna-se uma possibilidade de recurso didático.

O uso de jogos como objeto de aprendizagem é discutido em algumas pesquisas recentes. Nash (2005) cita que os jogos sérios consistem em um novo tipo de mídia de objetos de aprendizagem, incluídos em cursos presenciais ou online. Já, Torrente et al (2009), apresentam a plataforma $e$-Adventure, que permite o desenvolvimento de jogos sérios como objetos de aprendizagem. Entretanto, as especificações para metadados 
anteriormente mencionadas permitem a descrição de objetos de aprendizagem baseados em texto, imagem, áudio e vídeo: elas não podem ser diretamente utilizadas para descrever jogos sérios como recursos didáticos [Teixeira et al. 2007]. Isto se deve ao fato de que quando o jogo é usado como recurso didático, ele difere substancialmente dos outros tipos de mídia [Hendrix et al. 2012]. Uma solução de especificação de metadados proposta é trazida pro Hendrix et al. (2012). Ela baseia-se nos modelos existentes, tais como IEEE LOM, Sharable Content Object Reference Model (SCORM) e IMS Learning Design (IMS-LD). Além disto, os autores consideram os frameworks para avaliação de jogos sérios, tais como o Four Dimensional Framework. Entretanto, os mesmos não apresentam como os elementos podem ser incluídos nas especificações atuais, nem como implementar em ferramentas e/ou repositórios. O esquema de metadados proposto pelos autores permite uma série de comentários, além de permitir especificações técnicas e descritivas, por isso, neste tipo de metadado é feita uma distinção entre essas áreas técnicas e descritivas por um lado e o conjunto de campos de revisão do outro lado. A fim de contribuir com as pesquisas como as de Hendrix et al (2012), é que o projeto apresentado neste artigo foi desenvolvido.

\section{Metodologia}

O desenvolvimento deste trabalho caracteriza-se como uma pesquisa quantitativa aplicada sob a perspectiva exploratória, cujo objetivo era identificar os metadados necessários para a descrição de jogos sérios como objetos de aprendizagem. Portanto, por se tratar de uma investigação recente, foi necessária a abordagem exploratória, utilizandose de documentos e das descrições de jogos a fim de se verificar características comuns. Ainda, justifica-se como uma avaliação quantitativa e aplicada por se tratar de uma análise de 66 jogos cujo resultado será a aplicação. A condução da pesquisa ocorreu através das seguintes etapas: (a) seleção dos jogos sérios que poderiam ser caracterizados como objetos de aprendizagem; (ii) formulação de um questionário de avaliação; (iii) avaliação dos jogos através de um conjunto de questões; (iv) identificação de quais metadados existentes no OBAA que podem descrever os jogos sérios e (v) identificação de novos metadados, para uma descrição complementar.

Houveram dois tipos de jogos avaliados, aqui denominados de jogos baseados em internet e jogos convencionais. Os baseados em internet consistem em atividades grátis criadas com o intuito de repassar conhecimento, extremamente simples (simples de serem criados e simples de serem utilizados) e diretos, os quais são utilizados em um computador convencional ou dispositivo móvel. Já os jogos convencionais são aqueles que, tradicionalmente, são mais complexos e requerem artefatos específicos à sua execução (como um aparelho de videogame). Os avaliadores exploraram os jogos baseados em internet e, em seguida, respondiam ao questionário. Já para os jogos convencionais houve pesquisa utilizando-se da ferramenta de busca Google e vídeos encontrados no YouTube, devido a ausência dos aparelhos dos diversos fabricantes. A escolha dos jogos baseou-se naqueles que apresentavam mais popularidade nos repositórios ou sites especializados de jogos (Makuch, 2011; Rubenstein, 2013; Nintendo, 2013; Goldfarb, 2014; Tmcnet, 2005; Blizzard, 2013; Ltucker, 2010; Long, 2014).

A avaliação dos jogos foi realizada através de um conjunto de questões. Elas foram elaboradas com base na proposta Glover (2013). O autor defende que todo jogo tem seus objetivos alcançados através do conhecimento do usuário ou da capacidade motora do usuário de realizar a atividade, bem como que todo jogo possui um mecanismo 
de recompensa, sendo "Leaderboards", "Prizes" e "Achievements" as categorias mais evidentes. Além disso, o autor aborda o acompanhamento de progresso como característica importante no processo de aprendizagem, seja como feedback ao aluno, ou como forma de planejamento com o intuito de alcançar a próxima meta. Assim, originouse os pontos apresentados na Tabela 1.

Tabela 1: Questões para realização da avaliação dos jogos sérios.

\begin{tabular}{|c|c|}
\hline Ponto a ser avaliado & Detalhes sobre o ponto a ser avaliado \\
\hline 1. Game & $\begin{array}{l}\text { Título do jogo com link para sua utilização (OA) ou para vídeo demonstrativo } \\
\text { do jogo (jogo convencional). }\end{array}$ \\
\hline 2. Níveis ou Fases? & $\begin{array}{l}\text { O jogo funciona com sistema de níveis ou fases? A maioria dos jogos o usuário } \\
\text { progride através de uma série de atividades. Se essa série de atividades aumenta } \\
\text { gradativamente de dificuldade, o jogo é baseado em nível, se existe progresso } \\
\text { através da série mas a dificuldade é constante, o jogo é baseado em fases. }\end{array}$ \\
\hline $\begin{array}{l}\text { 3. Nível de conhecimento do } \\
\text { usuário }\end{array}$ & $\begin{array}{l}\text { Quanto de conhecimento sobre o assunto tratado no jogo o usuário deve ter para } \\
\text { conseguir realizar as atividades? Possíveis respostas são: nenhum (pode-se } \\
\text { alcançar os objetivos do jogo sem conhecimento algum sobre o assunto), baixo } \\
\text { (deve-se ter noção básica do assunto), razoável (é necessário conhecer o } \\
\text { assunto) e alto (o usuário só conseguirá alcançar os objetivos se possuir } \\
\text { conhecimento elevado sobre o assunto). }\end{array}$ \\
\hline 4. Pause? & O jogo oferece a funcionalidade de parar a atividade durante seu andamento? \\
\hline 5. Selecão de dificuldade? & O jogo permite alterar a dificuldade das atividades? \\
\hline 6. Foco em objetivo & O jogo foi feito de modo que o usuário consegue realizar as atividades com base \\
\hline $\begin{array}{l}\text { 6.1. Foco no conhecimento do } \\
\text { usuário? }\end{array}$ & $\begin{array}{l}\text { nos seus conhecimentos do assunto, na sua capacidade motora ou ambos? } \\
\text { Obs.: se no item } 3 \text { foi especificado "nenhum", o item 6.1. terá o valor padrão }\end{array}$ \\
\hline $\begin{array}{l}\text { 6.2. Foco na performance do } \\
\text { usuário? }\end{array}$ & NA. \\
\hline $\begin{array}{l}\text { 7. Recompensa } \\
\text { 7.1. Ranking? }\end{array}$ & $\begin{array}{l}\text { Existe uma lista online para todos os usuários verem qual o jogador que teve o } \\
\text { melhor progresso nas atividades? }\end{array}$ \\
\hline 7.2. Prêmio & O usuário recebe, ao finalizar uma tarefa, novas customizações para seu \\
\hline 7.2.1. Customização? & personagem no jogo (como roupas ou acessórios, por exemplo) ou desbloqueia \\
\hline 7.2.2. Desbloqueios? & novos itens, mapas, personagens, etc? \\
\hline 7.2.3. Conquistas? & $\begin{array}{l}\text { O jogo apresenta algum sistema de conquistas que encoraja os usuários a } \\
\text { realizar as atividades? }\end{array}$ \\
\hline $\begin{array}{l}\text { 8. Acompanhamento do } \\
\text { progresso? }\end{array}$ & $\begin{array}{l}\text { É possível acompanhar o progresso do usuário através daquela atividade ou do } \\
\text { jogo no geral? }\end{array}$ \\
\hline $\begin{array}{l}\text { 9. Pontuação } \\
\text { 9.1. Existe pontuação? } \\
\text { 9.2. A pontuação é o objetivo } \\
\text { do jogo? }\end{array}$ & $\begin{array}{l}\text { Existe algum sistema de pontuação no jogo? Ter a pontuação mais alta é o } \\
\text { objetivo principal do jogo? }\end{array}$ \\
\hline $\begin{array}{l}\text { 10. Multiplayer } \\
\text { 10.1. Versus? } \\
\text { 10.2. Cooperativo? }\end{array}$ & $\begin{array}{l}\text { É possível mais de um usuário jogar ao mesmo tempo? Cooperativo significa } \\
\text { que os usuários se ajudam para realizar as atividades. Versus é quando os } \\
\text { usuários competem entre si. }\end{array}$ \\
\hline 11. Tempo e/ou rodadas? & $\begin{array}{l}\text { O jogo tem um tempo fixo ou quantidade de rodadas (vezes que um usuário } \\
\text { pode jogar) especificado para realizar as atividades? }\end{array}$ \\
\hline 12. Som é dispensável? & O som é necessário para realizar as atividades? \\
\hline
\end{tabular}

\section{Análise e Resultados}

Após a formulação das questões e escolha dos jogos, deu-se início ao processo de análise dos mesmos. O processo de análise envolveu a verificação das características de 66 jogos, sendo 56 jogos baseados em internet e 10 jogos convencionais (2 para PlayStation 3, 2 para Xbox 360, 2 para Wii, 2 para PC e 2 para dispositivos móveis). Os jogos avaliados contemplavam diferentes tipos de mídias, áreas de conhecimento, público-alvo e duração. Ainda, cabe ressaltar que assim como existe uma diversidade de recursos didáticos, o mesmo se aplica ao contexto dos jogos. Há diversas opções, tanto focada no entretenimento quanto com objetivos "sérios". Contudo, a presente pesquisa não ficou restrita apenas aos jogos denominados sérios, pois se analisados sob a perspectiva e princípios dos objetos de aprendizagem, todo e qualquer recurso pode ser utilizado no 
contexto escolar, desde que sua aplicação seja para apoio ao ensino. Um resumo dos resultados é exibido na Tabela 2. De forma geral, percebe-se que os jogos selecionados têm como característica principal o foco no conhecimento do usuário, bem como o acompanhamento de seu progresso. Ambas características são imprescindíveis se for considerado que o objetivo de um jogo sério como objeto de aprendizagem é desenvolver um determinado conhecimento, e para isto, é importante oferecer um meio do usuário verificar sua evolução.

Tabela 2: Resumo dos resultados da avaliação.

\begin{tabular}{ll}
\hline \multicolumn{1}{c}{ Critério avaliado } & \multicolumn{1}{c}{ Resumo dos Resultados } \\
\hline Níveis ou Fases? & $41 \%$ níveis $(27)$ \\
& $39 \%$ fases $(26)$ \\
Nível de conhecimento do usuário & $20 \%$ nenhum $(13)$ \\
& $35 \%$ alto conhecimento $(23)$ \\
& $27 \%$ conhecimento razoável (18) \\
& $17 \%$ baixo conhecimento (11) \\
& $21 \%$ não requerem $(14)$ \\
Pause? & $17 \%$ possuem $(11)$ \\
& $18 \%$ não possuem $(12)$ \\
& $65 \%$ não aplicável (43) \\
Seleção de dificuldade? & $14 \%$ oferece $(9)$ \\
Foco no conhecimento do usuário? & $77 \%$ requer $(51)$ \\
Foco na performance do usuário? & $36 \%$ requer capacidade motora (24) \\
Ranking? & $14 \%$ possuem $(9)$ \\
Customização? & $9 \%$ possuem $(6)$ \\
Desbloqueios? & $38 \%$ possuem $(25)$ \\
Conquistas? & $12 \%$ possuem $(8)$ \\
Acompanhamento do progresso? & $92 \%$ é aplicável $(61)$ \\
Existe pontuação? & $62 \%$ oferece $(41)$ \\
A pontuação é o objetivo do jogo? & $15 \%$ sim $(10)$ \\
Multiplayer versus? & $6 \%$ sim (4) \\
Multiplayer cooperativo? & $8 \%$ sim $(5)-$ entretanto oferecem as possibilidades cooperativo e \\
& versus simultaneamente \\
Tempo e/ou rodadas? & $25 \%$ oferecem $(23)$ \\
Som é dispensável? & $2 \%$ é imprescindível (1) \\
& embora $72 \%$ ofereçam o recurso (48) \\
\hline
\end{tabular}

Com base nos resultados obtidos e na experiência obtida através da avaliação dos diferentes jogos, foi possível perceber diferentes características. Ambas auxiliaram na compreensão das informações relevantes para uma descrição adequada dos jogos como objetos de aprendizagem, as quais geraram a proposta apresentada na sequência.

\section{Proposta de Extensão dos Metadados OBAA}

A proposta apresentada envolveu duas etapas: (i) modificações à especificação OBAA atual - Tabela 3 e (ii) adição de novos elementos - Tabela 4. Inicialmente, buscou-se utilizar os metadados existentes para a descrição dos jogos, tendo como objetivo minimizar as modificações a especificação original. Neste caso, as sugestões apresentadas visam a criação de novos valores de preenchimento, sem a necessidade de alteração dos campos atuais. Por exemplo, os elementos tipo e nome tiveram a adição dos valores "vídeo game console" e "xbox, wii e playstation", respectivamente. 
Tabela 3: Modificações ao OBAA: modificações às categorias já existentes. Obs.: usa-se "?" para identificar que aquele item não pertence ao OBAA, logo, não possui número.

\begin{tabular}{|c|c|c|c|}
\hline Elemento & Descrição & Como preencher & $\begin{array}{l}\text { Motivo da } \\
\text { modificação }\end{array}$ \\
\hline 4.4.1.1. Type & $\begin{array}{l}\text { Tipo de } \\
\text { tecnologia } \\
\text { requerida para } \\
\text { usar o objeto de } \\
\text { aprendizagem }\end{array}$ & $\begin{array}{l}\text { operating system, browser, video game } \\
\text { console }\end{array}$ & $\begin{array}{l}\text { Recebe novo valor "video } \\
\text { game console" para } \\
\text { identificar jogos }\end{array}$ \\
\hline 4.4.1.2. Name & $\begin{array}{l}\text { Nome } \quad \text { da } \\
\text { tecnologia } \\
\text { requerida }\end{array}$ & $\begin{array}{l}\text { pc-dos, ms-windows, macos, unix, multi- } \\
\text { os, netscape, none, any, amaya, ms-internet } \\
\text { explorer, opera, communicator, xbox, wii, } \\
\text { playstation }\end{array}$ & $\begin{array}{l}\text { Recebe novos valores para } \\
\text { identificar os tipos de } \\
\text { videogames, "wii" } r \text { como } \\
\text { "xbox", " } \\
\text { "playstation" }\end{array}$ \\
\hline $\begin{array}{l}\text { 4.8. Supported } \\
\text { Platforms }\end{array}$ & $\begin{array}{l}\text { Plataformas } \\
\text { suportadas }\end{array}$ & mobile, dtv, web, console & $\begin{array}{l}\text { Recebe o item "console" } \\
\text { para } \\
\text { videogames }\end{array}$ \\
\hline $\begin{array}{l}\text { 4.9.5.1.2. } \\
\text { Specific Name }\end{array}$ & $\begin{array}{l}\text { Nome } \\
\text { tecnologia } \\
\text { requerida }\end{array}$ & $\begin{array}{l}\text { pc-dos, mswindows, } \\
\text { macos, unix, multi-os, none } \\
\text { mozillafirefox, any, arib, googlechrome, } \\
\text { applesafari, netscapecomunicator, amaya, } \\
\text { ms-internetexplorer, opera ginga, mhp, } \\
\text { davic, dase, gem, wii, playstation, xbox }\end{array}$ & $\begin{array}{l}\text { Recebe novos valores para } \\
\text { identificar os tipos de } \\
\text { videogames, "wii" } r \text { como } \\
\text { "xbox", } \\
\text { "playstation" }\end{array}$ \\
\hline $\begin{array}{l}\text { 5.2. Learning } \\
\text { Resource Type }\end{array}$ & $\begin{array}{l}\text { Tipo de recurso } \\
\text { de aprendizagem } \\
\text { utilizado, por } \\
\text { ordem de } \\
\text { predominância }\end{array}$ & $\begin{array}{l}\text { exercise, simulation, figure, diagram, } \\
\text { statement, index, } \\
\text { questionnaire, graph, slide, table, } \\
\text { selfassessment, exam, narrative text, } \\
\text { experiment, problem, lecture, game }\end{array}$ & $\begin{array}{l}\text { Recebe o valor "game" } \\
\text { para } \\
\text { videogames }\end{array}$ \\
\hline 4.4.1.1. Type & $\begin{array}{l}\text { Tipo de } \\
\text { tecnologia } \\
\text { requerida para } \\
\text { usar o objeto de } \\
\text { aprendizagem }\end{array}$ & $\begin{array}{l}\text { operating system, browser, video game } \\
\text { console }\end{array}$ & $\begin{array}{l}\text { Recebe novo valor "video } \\
\text { game console" para } \\
\text { identificar jogos }\end{array}$ \\
\hline 4.4.1.2. Name & $\begin{array}{l}\text { Nome } \\
\text { tecnologia } \\
\text { requerida }\end{array}$ & $\begin{array}{l}\text { pc-dos, ms-windows, macos, unix, multi- } \\
\text { os, netscape, none, any, amaya, ms-internet } \\
\text { explorer, opera, communicator, xbox, wii, } \\
\text { playstation }\end{array}$ & $\begin{array}{l}\text { Recebe novos valores para } \\
\text { identificar os tipos de } \\
\text { videogames, "wii, } r \text { como } \\
\text { "xbox", } \\
\text { "playstation" }\end{array}$ \\
\hline $\begin{array}{l}\text { 4.8. Supported } \\
\text { Platforms }\end{array}$ & $\begin{array}{l}\text { Plataformas } \\
\text { suportadas }\end{array}$ & mobile, dtv, web, console & $\begin{array}{l}\text { Recebe o item "console" } \\
\text { para } \\
\text { videogames }\end{array}$ \\
\hline $\begin{array}{l}\text { 4.9.5.1.2. } \\
\text { Specific Name }\end{array}$ & $\begin{array}{l}\text { Nome } \\
\text { tecnologia } \\
\text { requerida }\end{array}$ & $\begin{array}{l}\text { pc-dos, mswindows, } \\
\text { macos, unix, multi-os, none } \\
\text { mozillafirefox, any, arib, googlechrome, } \\
\text { applesafari, netscapecomunicator, amaya, } \\
\text { ms-internetexplorer, opera ginga, mhp, } \\
\text { davic, dase, gem, wii, playstation, xbox }\end{array}$ & $\begin{array}{l}\text { Recebe novos valores para } \\
\text { identificar os tipos de } \\
\text { videogames, "wii" } r \text { eomo } \\
\text { "xbox", "playstation" }\end{array}$ \\
\hline $\begin{array}{l}\text { 5.2. Learning } \\
\text { Resource Type }\end{array}$ & $\begin{array}{l}\text { Tipo de recurso } \\
\text { de aprendizagem } \\
\text { utilizado, por } \\
\text { ordem de } \\
\text { predominância }\end{array}$ & $\begin{array}{l}\text { exercise, simulation, figure, diagram, } \\
\text { statement, index, } \\
\text { questionnaire, graph, slide, table, } \\
\text { selfassessment, exam, narrative text, } \\
\text { experiment, problem, lecture, game }\end{array}$ & $\begin{array}{l}\text { Recebe o valor "game" } \\
\text { para } \\
\text { videogames }\end{array}$ \\
\hline
\end{tabular}

Na sequência, foram propostos elementos novos com o objetivo de complementar a descrição dos jogos sérios (Tabela 4). Para isto, foram propostas duas categorias: A. Mecanismos de recompensa e B. Objetivos. Ambas poderiam ser adicionadas a um metadados "Games", embora tais características não se limitem ao contexto de jogos. 
Tabela 4: Modificações ao OBAA: modificações às categorias já existentes. Obs.: usa-se "?" para identificar que aquele item não pertence ao OBAA, logo, não possui número. Elemento Descrição

Como
Motivo da modificação preencher

$\begin{array}{lll}\begin{array}{l}\text { A. Reward } \\ \text { Mechanisms }\end{array} & \begin{array}{l}\text { Sistema de recompensas } \\ \text { do serious game }\end{array} & \begin{array}{l}\text { Nova categoria que trata do sistema de } \\ \text { recompensa daquele jogo (sistema não existente } \\ \text { em objetos de aprendizagem tradicionais) }\end{array} \\ \begin{array}{l}\text { A.1. } \\ \text { Leaderboards }\end{array} & \begin{array}{l}\text { Determina se a true, false } \\ \text { recompensa é em forma } \\ \text { de ranking de pontuação } \\ \text { dos usuários }\end{array} & \begin{array}{l}\text { Determina se a recompensa é em forma de } \\ \text { ranking de pontuação dos usuários. Rankings } \\ \text { exibem, para todos, os usuários com melhor } \\ \text { desempenho no jogo } \\ \text { Determina se a recompensa é em forma de } \\ \text { prêmios para os usuários (por exemplo, dinheiro } \\ \text { vetermina se a - }\end{array} \\ \begin{array}{l}\text { a.2. Prizes } \\ \text { de prêmios por completar alguma fase) }\end{array}\end{array}$

A.2.1. usuários

Customizations

Determina se o tipo de prêmio recebido é customização

A.2.2. Unlocks Determina se o tipo de prêmio recebido é desbloqueio

A.3. Determina se a Achievements recompensa é em forma de conquistas para os usuários

B.?. Goals Define como o objetivo da atividade pode ser alcançado

B.?. Progress O jogo possui tracking acompanhamento de progresso

B.?. Difficulty A dificuldade das change atividades pode ser modificada

B.?. Time / O usuário tem tempo ou rounds quantidade de rodadas para finalizar a tarefa

B.?. Pause A atividade pode ser parada e retomada de onde parou

B.?. Levels or Define se o jogo é stages

baseado em níveis ou em fases

\section{B.x. Score \\ Pontuação}

B.x.1. HasScore

Possui pontuação

B.x.2. Score is A pontuação é o objetivo

true, false

true, false

true, false

true, false

true, false both

true, false

true, false

true, false

true, false knowledge,

performance,

levels, stages

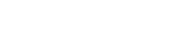
goal

A fim de exemplificar o uso dos metadados proposto, foi selecionado o jogo Minecraft, cujas características são apresentadas na Figura 2. Para isto, utilizou-se o conjunto de metadados OBAA Lite (apenas os metadados obrigatórios), adicionando-se os elementos necessários para a descrição do jogo.
Subitem de "Prizes" que especifica se o prêmio recebido é algum tipo de customização (como roupas ou itens para o personagem do game) Subitem de "Prizes" que especifica se o prêmio recebido é algum tipo de desbloqueio (como novas fases, mapas, personagens, itens, etc) Determina se a recompensa é em forma de conquistas para os usuários (por exemplo, uma medalha para exibir no perfil)

"knowledge" se o objetivo depende do conhecimento do usuário, "performance" se é da capacidade física do usuário em realizar a tarefa ou "both" caso dependa dos dois Conforme coletado durante a análise, há a necessidade para a criação deste item

Conforme coletado durante a análise, há a necessidade para a criação deste item

Conforme coletado durante a análise, há a necessidade para a criação deste item

Conforme coletado durante a análise, há a necessidade para a criação deste item

Este item pode ser ainda subdivido a fim de detalhar como o sistema do jogo funciona (se há uma sequência lógica de níveis, se obrigatoriamente deve-se seguir esta sequência, é textual e outra fase contém informações em áudio, por exemplo), etc)

É necessário um item que defina se o jogo possui algum tipo de mecanismo de pontuação e se essa pontuação é o objetivo do usuário

$-$ se as fases mudam a mecânica do jogo (uma fase 


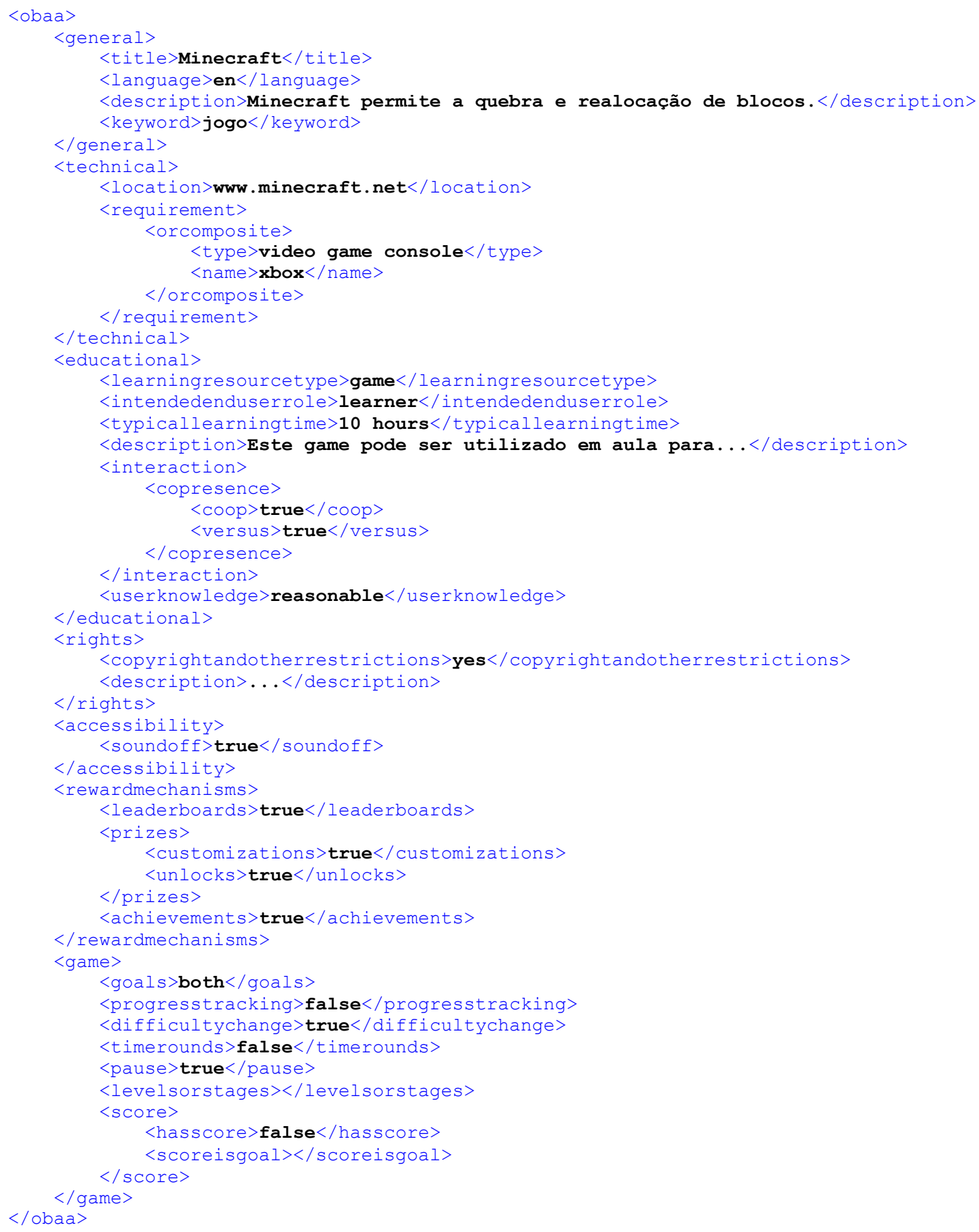

Figura 1. Exemplo de código gerado com os metadados propostos aplicados na versão reduzida do OBAA

\section{Conclusões}

A partir da perspectiva que os jogos sérios têm como um de seus objetivos a aprendizagem ou treinamento (simulação) de algum conteúdo, é almejável que ele seja tratado como um objeto de aprendizagem. Desta forma, os jogos sérios devem ser considerados pelas tecnologias envolvidas, como repositórios e arquivos de metadados. Neste sentido, o presente trabalho buscou investigar quais informações são necessárias para a descrição 
de um jogo sério como objeto de aprendizagem. Para isto, foram avaliados 66 jogos e, ao final, apresentada uma proposta de metadados aderente à especificação OBAA. Também foi apresentado um exemplo de como ficaria um arquivo de metadados a partir de um jogo disponível no mercado, demonstrando assim a viabilidade de utilização dos elementos sugeridos.

Inicialmente buscou-se reaproveitar os campos já existentes, adaptando-os ao contexto de jogos. Logo, as principais sugestões ocorreram nos itens referentes a descrição técnica: tipo e nome da tecnologia utilizada, plataformas suportadas. Também sugeriu-se o acréscimo do valor "jogo" no item de tipos de recursos de aprendizagem.

Adicionalmente, foram indicados outros elementos a serem acrescidos em uma categoria própria sobre jogos. Nela, poderiam ser informados dados sobre os mecanismos de recompensas (prêmios, desbloqueios, conquistas) e objetivos (progresso, dificuldades, etapas, pausa e pontuação). Optou-se por não determinar um posicionamento específico dentro da especificação do OBAA, por este trabalho se tratar de uma proposta. E, portanto, cabe aos mantenedores da especificação avaliar e estabelecer um local apropriado.

O trabalho destaca-se por ter conduzido uma exaustiva avaliação, envolvendo um número significativo de jogos. Além de utilizar-se de pesquisas anteriores como fundamento da proposta apresentada. Como limitação, pode-se descrever a ausência de equipamentos para avaliação dos jogos denominados convencionais. Buscou-se informações através dos sites dos fornecedores, entretanto, sabe-se que em alguns casos as informações podem não terem sido apresentadas adequadamente, o que geraria interpretação equivocada das suas potencialidades.

Como trabalhos futuros têm-se a apresentação da proposta aos responsáveis pelo OBAA. Caso aceito, o mesmo poderá ser aplicado nos repositórios e ferramentas existentes que são aderentes a especificação. Com isto, ampliam-se as possibilidades pedagógicas do uso de jogos com fins didáticos.

\section{Referências}

Blizzard Entertainment (2013) "Blizzard Entertainment Statistics". Disponível em: http://www.statisticbrain.com/blizzard-entertainment-statistics/, acessado em jan. de 2014.

Glover, I. (2013) "Play As You Learn: Gamification as a Technique for Motivating Learner", Proceedings of World Conference on Educational Multimedia, Hypermedia and Telecommunications.

Goldfarb, A. (2014) "GTA 5 Has Shipped 32.5 Million Copies". Disponível em: http://www.ign.com/articles/2014/02/03/gta-5-has-shipped-325-million-copies, acessado em fev. de 2014.

Hendrix, M. et al. (2012) "Defining a Metadata Schema for Serious Games as Learning Objects". 4th International Conference on Mobile, Hybrid, and On-line Learning (eLmL 2012).

Janson, A. Janson, R. (2009) "Integrating Digital Learning Objects in the classroom: a need for educational leardship: the benefits and challenges of using Digital Learning Objects in the classroom”. Disponível em http://researchcommons.waikato.ac.nz/ 
bitstream/handle/10289/5424/Digital\%20learning\%20objects.pdf?sequence $=1$, acessado em jun. 2014.

Long, N. (2014) "Two billion downloads? We're just getting started, says Angry Birds creator Rovio". Disponível em: http://www.edge-online.com/features/two-billiondownloads-were-just-getting-started-says-angry-birds-creator-rovio/, acessado em fev. de 2014.

Ltucker. (2010) “Tetris Game Surpasses 100 Million Paid Mobile Downloads, Is the BestSelling Mobile Phone Game of All Time". Disponível em: http://www.ea.com/news/tetris-game-surpasses-100-million-mobile-downloads, acessado em jan. de 2014.

Makuch, E. (2014) “Call of Duty: Black Ops sells 29 million - Analyst. 2011”. Disponível em: http://www.gamespot.com/articles/call-of-duty-black-ops-sells-20-millionanalyst/1100-6312133/, acessado em jan. de 2014.

Morais, A. M. (2011) "Planejamento e desenvolvimento de um serious game voltado ao ensino de saúde bucal em bêbes". Dissertação de Mestrado. Programa de PósGraduação em Modelos de Decisão e Saúde. Universidade Federal da Paraíba.

Nash, S. (2005) "Learning Objects, Learning Object Repositories, and Learning Theory: Preliminary Best Practices for Online Courses", Interdisciplinary Journal of Knowledge and Learning Objects. Vol 1.

Nintendo (2013) “Top Selling Software Sales Unit - Wii”. Disponível em: http://www.nintendo.co.jp/ir/en/sales/software/wii.html. Acessado em jan. de 2014.

Rubenstein, J. (2013) “That's a Lot of Blocks: Minecraft Creeps Past 10 Million on Xbox 360” Disponível em: http://news.xbox.com/2013/12/games-minecraft-announcement, acessado em jan. de 2014.

Susi, T.; Johannesson, M.; Backlund, P. (2005) "Serious Games: an overview, Technical Report HS-IKI-TR-07-001". Disponível em t: http://www.autzones.com/din6000/ textes/semaine12/SusiEtAl(2005).pdf, acessado em jul. 2014.

Teixeira, J. S. F., Sá, E. J., Fernandes, C. T. (2007) "Representação de Jogos Educacionais a partir do Modelo de Objetos de Aprendizagem". XIII Workshop sobre Informática na Escola, Anais do XXVII Congresso da SBC, Rio de Janeiro, RJ.

Tmcnet (2005) "The Sims Franchise Celebrates Its Fifth Anniversary and Continues to Break Records". Disponível em: http://www.tmcnet.com/usubmit/2005/feb/ 1114806.htm, acessado em jan. de 2014.

Torrente, J., Moreno-Ger, P., Martínez-Ortiz, I., Fernandez-Manjon, B. (2009) "Integration and Deployment of Educational Games in e-Learning Environments: The Learning Object Model Meets Educational Gaming”, Educational Technology \& Society, vol. 12.

Vicari, R. M. et al. (2010) "Brazilian proposal for agent-based learning objects metadata standard-OBAA". Metadata and Semantic Research. Springer Berlin Heidelberg. 